\title{
Inflexible Functional Connectivity of the Dorsal Anterior Cingulate Cortex in Adolescent Major Depressive Disorder
}

\author{
Tiffany C Ho*,1,2,10, Matthew D Sacchet ${ }^{2,3,10}$, Colm G Connolly', Daniel S Margulies ${ }^{4}$, Olga Tymofiyeva ${ }^{5}$, \\ Martin P Paulus ${ }^{6,7}$, Alan N Simmons ${ }^{7,8}$, lan H Gotlib ${ }^{2,9}$ and Tony T Yang' \\ 'Division of Child and Adolescent Psychiatry, Department of Psychiatry, University of California, Weill Institute for Neurosciences, San Francisco, CA, \\ USA; 'Department of Psychology, Stanford University, Stanford, CA, USA; ${ }^{3}$ Department of Psychiatry and Behavioral Sciences, Stanford University, \\ Stanford, CA, USA; ${ }^{4}$ Max Planck Institute, Human Cognitive and Brain Sciences, Leipzig, Germany; ${ }^{5}$ Department of Radiology, University of \\ California, San Francisco, CA, USA; ${ }^{6}$ The Laureate Institute for Brain Research, Tulsa, OK, USA; ' Department of Psychiatry, University of California, \\ San Francisco, CA, USA; ${ }^{8}$ Center of Excellence for Stress and Mental Health, Veterans Affairs San Diego Health Care System, San Diego, CA, USA; \\ ${ }^{9}$ Neurosciences Program, Stanford University, Stanford, CA, USA
}

Recent evidence suggests that anterior cingulate cortex (ACC) maturation during adolescence contributes to or underlies the development of major depressive disorder (MDD) during this sensitive period. The ACC is a structure that sits at the intersection of several task-positive networks (eg, central executive network, CEN), which are still developing during adolescence. While recent work using seed-based approaches indicate that depressed adolescents show limited task-evoked vs resting-state connectivity (termed 'inflexibility') between the ACC and task-negative networks, no study has used network-based approaches to investigate inflexibility of the ACC in task-positive networks to understand adolescent MDD. Here, we used graph theory to compare flexibility of network-level topology in eight subregions of the ACC (spanning three task-positive networks) in 42 unmedicated adolescents with MDD and 53 well-matched healthy controls. All participants underwent $\mathrm{MMRI}$ scanning during resting state and a response inhibition task that robustly engages task-positive networks. Relative to controls, depressed adolescents were characterized by inflexibility in local efficiency of a key ACC node in the CEN: right dorsal anterior cingulate cortex/medial frontal gyrus (R dACC/MFG). Furthermore, individual differences in flexibility of local efficiency of R dACC/MFG significantly predicted inhibition performance, consistent with current literature demonstrating that flexible network organization affords successful cognitive control. Finally, reduced local efficiency of dACC/MFG during the task was significantly associated with an earlier age of depression onset, consistent with prior work suggesting that MDD may alter functional network development. Our results support a neurodevelopmental hypothesis of MDD wherein dysfunctional self-regulation is potentially reflected by altered ACC maturation.

Neuropsychopharmacology (2017) 42, 2434-2445; doi:I0.1038/npp.20 17.103; published online 19 July 2017

\section{INTRODUCTION}

Major depressive disorder (MDD) is a highly debilitating disorder with enormous personal costs and societal burden. MDD often first emerges during adolescence (Kessler et al, 2005), a sensitive period of brain development coinciding with significant changes in physical and social development (Blakemore and Choudhury, 2006). Brain maturation during adolescence includes not only structural changes such as increased myelination and changes in gray matter density, but also functional changes that occur at the level of network connectivity and organization (Fair et al, 2007a, 2009; Power

*Correspondence: Dr TC Ho, Department of Psychology, Stanford University, 450 Serra Mall, Jordan Hall Room \#169, Stanford, CA 94305, USA, Tel: + I 510520 909I, Fax: 6507255699 ,

E-mail: tiffnie@stanford.edu

${ }^{10}$ These two authors contributed equally to this work.

Received 29 November 2016; revised I5 April 2017; accepted I4 May 2017; accepted article preview online 29 May 2017 et al, 2010). Therefore, examining the functional networks associated with MDD early in the course of the disorder, during this sensitive period of development, is important for determining how the developmental organization of functional brain networks is affected by the onset of depression.

The human brain is intrinsically organized into functional networks (Fox et al, 2005; Yeo et al, 2011), several of which have been investigated in the context of MDD (Kaiser et al, 2015a). These intrinsic functional networks (IFNs) include task-negative networks, such as the default mode network (DMN), and task-positive networks, such as the central executive network (CEN) and salience network (SN) (Menon, 2011). Studies on depression have increasingly focused on IFNs centered on the anterior cingulate cortex (ACC), a structure situated medially in the cerebral cortex and superior to the corpus callosum. The ACC exhibits diverse functioning -including conflict error monitoring, attentional orienting, affective processing, and behavioral selection (Bush et al, 2000) - and has been implicated in many psychopathologies 
(Menon, 2011). Importantly, the ACC can be subdivided into several regions, with distinct portions being connected to distinct IFNs (Margulies et al, 2007). The subgenual ACC (sgACC), for instance, has typically been reported to be connected with cortical limbic structures and the DMN, and is implicated in affective and self-referential processing (Hamilton et al, 2015). In contrast, the dorsal ACC (dACC) is connected with regions in the CEN and $\mathrm{SN}$ that are implicated in goal maintenance and the integration of sensory and visuomotor input to guide behavior (Seeley et al, 2007; Uddin, 2014). In the context of understanding adolescent development and vulnerability to MDD, compelling evidence indicates that ACC connectivity undergoes tremendous reorganization during adolescence (Fair et al, 2007a; Kelly et al, 2009; Power et al, 2010). Specifically, the sgACC becomes more strongly connected with the DMN over adolescent development, whereas the dACC is more tightly connected with the CEN during childhood but is then more strongly connected with the SN by adulthood (Fair et al, 2009; Power et al, 2010; Sole-Padulles et al, 2016; Uddin et al, 2011).

Given the role of the ACC in integrating multimodal inputs and orchestrating complex behavioral outputs and the maturational changes that occur during adolescence, researchers suggest that altered developmental changes in IFNs that stem from the ACC impact self-regulation processes, which then confer heightened risk of adolescent MDD (Lichenstein et al, 2016). Indeed, studies of restingstate functional connectivity in adolescent MDD have found that depression is associated with altered connectivity among ACC regions and the CEN, SN, and DMN (Connolly et al, 2013; Davey et al, 2012; Jacobs et al, 2014; Sacchet et al, 2016). Longitudinal changes in resting-state functional connectivity of the ACC have also been shown to predict the development of depressive symptoms during adolescence (Davey et al, 2014; Strikwerda-Brown et al, 2015), which suggests that altered developmental trajectories of IFNs anchored by the ACC may contribute to the development of MDD. Complementing these findings are task-based fMRI studies showing that hyperconnectivity of the DMN with $\mathrm{ACC}$ regions in the $\mathrm{CEN}$ and $\mathrm{SN}$ during active task processing is associated with an earlier age of depression onset (Ho et al, 2015). Furthermore, unlike their healthy peers, depressed adolescents fail to exhibit state-dependent changes (ie, between active task processing and resting state) in DMN-based connectivity with ACC regions in taskpositive IFNs. This absence of connectivity change as a function of brain state, herein termed 'inflexibility,' may be a neurodevelopmentally sensitive marker of MDD (Ho et al, 2015). Interpreted in light of recent work demonstrating that flexible configuration of task-positive IFNs subserves adaptive cognitive control (Cole et al, 2013, 2014a; Dwyer et al, 2014; Shine et al, 2016) and reflects developmental processes during adolescence (Hutchison and Morton, 2016), these findings support current theoretical models of ACC-based connectivity in the development of self-regulatory processes during adolescence (Lichenstein et al, 2016). Under this model, adolescent MDD is characterized by maladaptive or developmentally atypical inflexibility of ACC-based connectivity. However, previous studies have been limited in their examinations of state-dependent ACC-based functional connectivity of adolescents with MDD by assessing only connectivity during resting state, as well as relying on seed- based analyses rather than network-based approaches, which provide more comprehensive assessments of the topology and organization of IFNs (although see Sacchet et al, 2016).

To address this limitation, we used graph theory, a mathematical approach from network science that provides summary measures of network-level properties, to investigate ACC connectivity in unmedicated adolescents with MDD and well-matched healthy controls. The benefit of network-based approaches is that we gain an understanding of the topology of network organization and the importance of specific regions in the context of the entire brain as a network (Rubinov and Sporns, 2010). For example, with graph theory, we can quantify the degree of a region, which reflects the region's total number of connections, or its clustering coefficient, which quantifies local connectivity, or how tightly a region is connected with its neighbors. Similarly, local efficiency, which is the inverse distance connecting a region with its neighbors, is thought to provide insight into a region's involvement in information transfer. Indeed, such graph metrics have commonly been examined to understand the topology of resting-state networks in adults and adolescents with depression (Jin et al, 2011; Sacchet et al, 2016; Zhang et al, 2011, 2015). However, no studies to date have directly examined flexibility, or statedependent changes in connectivity, in the ACC to elucidate the neuropathophysiology of adolescent MDD.

Thus, in the present study, we computed local graph metrics (ie, degree, clustering coefficient, local efficiency) from eight subregions of the ACC that span major taskpositive IFNs. Specifically, we computed these graph metrics during resting state and during a response inhibition task that robustly engages the CEN and other task-positive IFNs. We identified which ACC regions exhibited topological properties that differed between groups as a function of change in brain state (rest $v s$ task). We hypothesized that, compared to healthy controls, depressed adolescents would show neural inflexibility of the ACC, operationalized by limited state-dependent changes in graph metrics of the ACC. Guided by previous literature demonstrating that statedependent changes in the topology of task-positive IFNs (specifically, CEN) relate to performance on demanding cognitive tasks in adolescents (Dwyer et al, 2014) and current theoretical models positing that adolescent MDD reflects altered neurodevelopmental trajectories of ACC connectivity underlying self-regulation (Lichenstein et al, 2016), we hypothesized that state-dependent changes (ie, flexibility) in ACC connectivity as measured by local graph metrics would be associated with successful task performance. Finally, based on prior work demonstrating that depressed adolescents with greater inflexibly elevated ACC connectivity with the DMN during active affective challenge endorsed greater severity of depressive symptoms and had an earlier age of depression onset (Ho et al, 2015), we hypothesized that network-level organization of task-evoked ACC connectivity would be associated with greater depressive severity and an earlier age of depression onset among the MDD group. 
Table I Demographic and Clinical Information

\begin{tabular}{|c|c|c|c|}
\hline & MDD & CTL & Statistic \\
\hline Final $n$ & 42 & 53 & \\
\hline Gender (M/F) & $17 / 25$ & $21 / 32$ & $\chi_{1}=0.05 ; p=0.82$ \\
\hline Age (years) & $16.0 \pm 1.3$ & $16.1 \pm 1.3$ & $t_{93}=-0.05 ; p=0.96$ \\
\hline Hollingshead & $40 \pm 38(11-73) *$ & $29 \pm 35.5(0-77)[1]^{*}$ & $W=|24| ; p=0.36$ \\
\hline Tanner Score & $4 \pm 0.5(3-5)^{*}$ & $4 \pm 1(3-5)^{*}$ & $W=1290 ; p=0.25$ \\
\hline CGAS & $64 \pm 12(4 \mid-90)$ & $90.7 \pm 6(75-100)$ & $t_{58.86}=-|3.32, p<0.00|$ \\
\hline CDRS-R & $70.2 \pm 9.5(44-85)$ & $33.6 \pm 6.1(30-61)$ & $t_{68.71}=21.82, p<0.001$ \\
\hline MASC & $58.9 \pm 9.4(34-83)$ & $42 \pm 8.5(25-6 I)[1]$ & $t_{85.58}=9.11, p<0.001$ \\
\hline RADS-2 & $65.5 \pm 8.7(4 \mid-87)$ & $41 \pm 7.3(30-56)[1]$ & $t_{82.1}=|4.66, p<0.00|$ \\
\hline Response time on non-missed GO trials & $777.78 \pm 20.04[5]$ & $769.52 \pm 21.33[8]$ & $F_{1,80}=0.08 ; p=0.78$ \\
\hline No comorbidities & $18(42.86 \%)$ & & \\
\hline GAD & $15(35.71 \%)$ & & \\
\hline PTSD & $2(4.76 \%)$ & & \\
\hline ADHD & $2(4.76 \%)$ & & \\
\hline Conduct disorder & | (2.38\%) & & \\
\hline Anxiety disorder not otherwise specified & | (2.38\%) & & \\
\hline PTSD+ADHD & $2(4.76 \%)$ & & \\
\hline GAD+ADHD+social phobia & I (2.38\%) & & \\
\hline
\end{tabular}

Abbreviations: $\chi^{2}, \chi^{2}$ test for equality of proportions; ADHD, attention deficit hyperactivity disorder; CDRS-R, Children's Depression Rating Scale-Revised; CGAS, Children's Global Assessment Scale; GAD, generalized anxiety disorder; IQR, interquartile range; MASC, Multidimensional Anxiety Scale for Children; PTSD, post traumatic stress disorder, RADS-2, Reynolds Adolescent Depression Scale 2nd edition; t, Student's $t$-test; W, Wilcoxon rank-sum test; WASI, Wechsler Abbreviated Scale of Intelligence.

Values are presented as mean \pm SEM (min-max) or median \pm IQR (min-max) if indicated by *. The optional number in brackets indicates the number of missing participants. Statistic: $W, \chi^{2}, t$ (when conditions of homogeneity of variance are met), or Welch's $t$-test (when conditions of homogeneity of variance are not met).

\section{MATERIALS AND METHODS}

\section{Participants and Clinical Information}

Sixty-three MDD and 70 healthy control (CTL) adolescents participated in this study. Eighteen MDD and 17 CTL participants were excluded for excessive motion during either the task or resting-state scans (see 'MRI Data Preprocessing' below and the Supplementary Information for more details). Three MDD participants were excluded for active antidepressant usage, given evidence that antidepressants can significantly alter functional connectivity patterns (Ho et al, 2015). Thus, we report results from 42 unmedicated adolescents with MDD and 53 CTL participants. Study protocol, recruitment procedures, clinical and diagnostic assessments, and inclusion/exclusion criteria have been previously described (Connolly et al, 2013; Ho et al, 2015; Sacchet et al, 2016) and are included in the 'Participants and Clinical Information' section of the Supplementary Information. Resting-state data from all participants in this report have been previously reported (Sacchet et al, 2016); we report fewer participants in the present study as our analyses necessitated that participants have both usable resting-state and task-based fMRI data.
Notably, in our prior investigation we used a different parcellation scheme with only 17 whole IFNs as nodes. In the present study, we used 51 spatially isolated regions comprising 7 IFNs as nodes and focused our examination of local graph metrics to regions of the ACC that span task-positive IFNs. Finally, we wish to note two previous seed-based connectivity studies wherein resting-state data from $18 \mathrm{MDD}$ and 31 CTL were previously reported (Connolly et al, 2013; Ho et al, 2015). Those studies focused only on connectivity patterns of regions based in the DMN and did not leverage network-based approaches. Critically, in the present study, we report novel fMRI and behavioral data from the response inhibition task and utilize graph theory to examine ACCbased topology as a function of brain state (ie, neural flexibility).

Participants were 13-18 years of age, right-handed, and neurologically and physically healthy. All participants and their parent(s) and/or legal guardian(s) provided written informed assent and consent, respectively, in accordance with the Declaration of Helsinki. The Institutional Review Boards at the University of California San Diego, Rady Children's Hospital in San Diego, the County of San Diego, and the University of California San Francisco approved 
this study. All potential MDD participants were administered the Schedule for Affective Disorders and Schizophrenia for School-Age Children-Present and Lifetime Version (KSADS$\mathrm{PL})$ to establish a DSM-IV diagnosis. All potential CTL participants were administered the Diagnostic Interview Schedule for Children (Shaffer et al, 2000) Diagnostic Predictive Scale (Lucas et al, 2001) to screen for the presence of any Axis I disorders. All participants completed several self-report and clinical assessments, including the self-report Reynolds Adolescent Depression Scale (RADS-2; Reynolds, 2002). See 'Participants and Clinical Information' in the Supplementary Information for details and Table 1 for more details.

\section{Task and Behavioral Data}

Participants performed a modified response inhibition task that has been shown to robustly activate regions in task-positive IFNs (Dodds et al, 2011) (see also 'Task Effects in Task-Positive Networks' in Supplementary Information). During the task, participants were instructed to make binary gender discriminations of faces using a two-finger button box but to withhold a response when presented with a stop tone delivered through their headphones. Participants were encouraged to respond as accurately and as quickly as possible. The task consisted of 292 trials (228 GO, 64 STOP); on half of the STOP trials, the stop tone occurred $150 \mathrm{~ms}$ after stimulus onset (EASY) and on the other half, $450 \mathrm{~ms}$ after stimulus onset (HARD). See 'Response Inhibition Task' in the Supplementary Information for more details. A repeatedmeasures ANOVA with group and difficulty modeled as factors was used to test for group differences in response time on GO trials and accuracy (percentage of successful stops). Five MDD and eight CTL participants did not have their behavioral responses properly recorded and were therefore excluded from all analyses requiring behavioral responses. Prior to their scan, all participants practiced the response inhibition task in a behavioral session. All statistical analyses were conducted in $R$ version 3.3.1 unless otherwise noted.

\section{MRI Acquisition}

All participants underwent a structural scan and two functional scans (resting state and task). See 'MRI Acquisition' in the Supplementary Information for details.

\section{MRI Data Preprocessing}

Data were preprocessed using AFNI (Cox, 1996) and FSL (Smith et al, 2004) software. T1-weighted images were skullstripped using AFNI's 3dSkullStrip and transformed into MNI152 space using FSL's FLIRT with subsequent nonlinear alignment using FNIRT. EPI data were despiked, slicetime and motion corrected, bandpass filtered, and smoothed using AFNI's preprocessing suite. Multiple linear regression was used to regress out effects of motion and physiological signals from each volume and volumes associated with excessive movement were censored. Individuals with more than $20 \%$ of their volumes exhibiting excessive motion in either the task or resting-state scans were excluded from all subsequent analysis (18 MDD and 17 CTL were excluded). See 'MRI Data Preprocessing' in the Supplementary Information for details. We further conducted control analyses to test for group differences in motion-related parameters. See 'Control Analyses for Movement and Censored Volumes' in the Supplementary Information for details.

\section{Network Node and Edge Definition}

Graphs are defined as sets of nodes and their edges (ie, connections between nodes). In this study, we defined nodes based on the 51 unique regions comprising the 7 Yeo networks (Yeo et al, 2011) (see also Figure 1 and Table 2). We defined edges as the strength of functional connections between pairs of nodes, operationalized using the absolute value of partial correlation coefficients (Sacchet et al, 2016; Zhang et al, 2011). See Figure 2 for a summary of the analysis pipeline.

\section{Global and Local Graph Metrics}

Global graph metrics summarize whole-graph properties. Local graph metrics characterize single nodes in a graph based on network characteristics related to that node. Global and local graph metrics were computed from binarized correlation matrices using an adaptive thresholding procedure. See 'Network Binarization' in the Supplementary Information for details. On the basis of previous work (Jin et al, 2011; Sacchet et al, 2016), our local graph metrics of interest included: (1) degree; (2) clustering coefficient, and (3) local efficiency. Although our hypotheses focused on

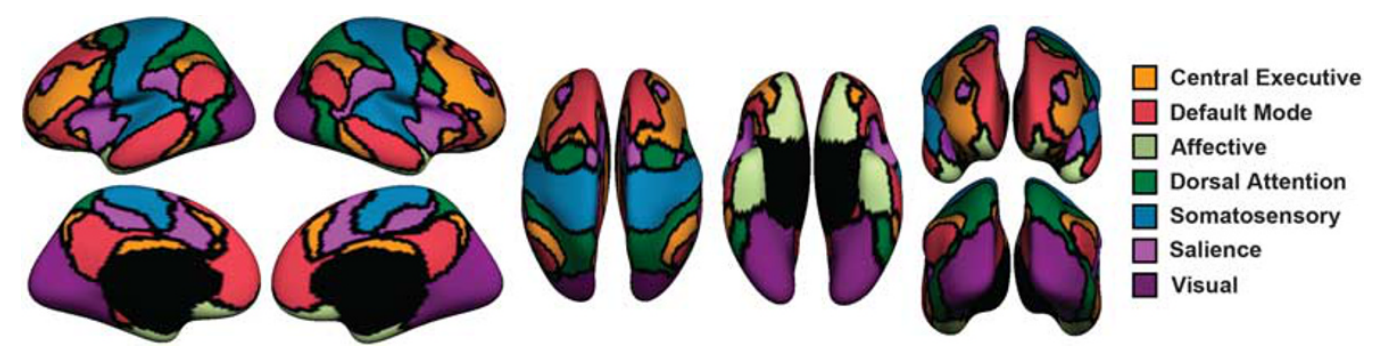

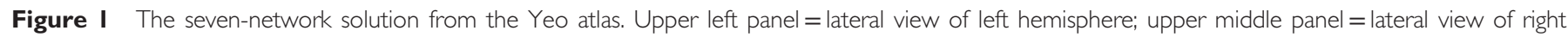

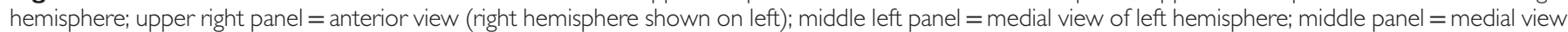

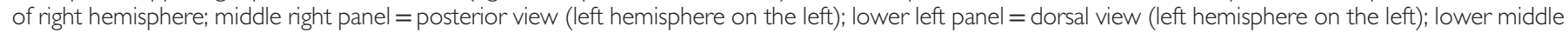

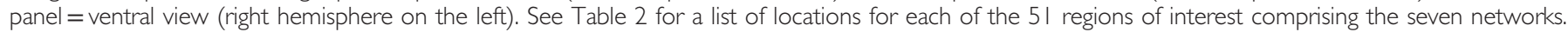


local graph metrics of the ACC, based on prior graph studies of MDD (Jin et al, 2011; Sacchet et al, 2016; Zhang et al, 2011), we also computed the global graph metrics of (1) clustering coefficient, (2) characteristic path length, (3) small-worldness, and (4) global efficiency. Graph analyses were conducted using the Brain Connectivity Toolbox

Table 2 Location of the 5 I Nodes Comprising the 7 Yeo Networks

\begin{tabular}{|c|c|c|c|c|}
\hline Region (Brodmann's areas) & $x$ & $y$ & $\mathbf{z}$ & Network \\
\hline L precentral/postcentral gyrus (BA 4/6) & -35 & -22 & 44 & Somatosensory \\
\hline L precentral gyrus, middle frontal gyrus (BA 6) & -26 & -4 & 55 & Dorsal attention \\
\hline L inferior frontal gyrus (BA 9) & -49 & 4 & 32 & Dorsal attention \\
\hline L postcentral gyrus, inferior parietal lobule (BA 40) & -59 & -32 & 28 & Salience \\
\hline L superior frontal gyrus (BA 9) & -29 & 42 & 31 & Salience \\
\hline L cingulate gyrus, superior frontal gyrus (BA 32)* & -6 & 12 & -40 & Salience \\
\hline L orbitofrontal cortex/subgenual anterior cingulate cortex (BA I| / 25$)^{*}$ & -12 & 36 & -21 & Limbic \\
\hline L parahippocampal cortex, uncus, temporal gyrus (BA 20/38) & -35 & -3 & -35 & Limbic \\
\hline$L$ inferior parietal lobule (BA 40) & -43 & -52 & 48 & CEN \\
\hline $\mathrm{L}$ insula, inferior frontal gyrus (BA 13/47) & -30 & 22 & -4 & CEN \\
\hline L precuneus (BA 7) & -3 & -71 & 46 & CEN \\
\hline$L$ cingulate (BA 32)* & -5 & -14 & 28 & CEN \\
\hline L dorsal anterior cingulate cortex/middle frontal gyrus (BA 8/6)* & -4 & 26 & 45 & CEN \\
\hline L angular gyrus/supramarginal gyrus (BA 39/40) & -47 & -62 & 33 & DMN \\
\hline L middle and inferior temporal gyrus (BA 21/22) & -56 & -16 & 15 & DMN \\
\hline L frontal gyrus (BA 10/9/47) & -14 & 44 & 28 & DMN \\
\hline L posterior cingulate cortex, precuneus (BA 23/3|/7) & -8 & -52 & 30 & DMN \\
\hline L parahippocampal gyrus, culmen & -21 & -29 & -20 & $\mathrm{DMN}$ \\
\hline R occipital cortex, fusiform gyrus (BA 17//8/19/36) & 25 & -74 & 3 & Visual \\
\hline R precentral and postcentral gyrus (BA 4/6) & 38 & -19 & 40 & Somatosensory \\
\hline R superior frontal gyrus, anterior cingulate cortex (BA 6, 32/24)* & 9 & 22 & 49 & Salience \\
\hline R orbitofrontal cortex/subgenual anterior cingulate cortex (BA I|/25)* & 13 & 38 & -21 & Limbic \\
\hline R parahippocampal cortex, uncus, temporal gyrus (BA 20/38) & 37 & -2 & -36 & Limbic \\
\hline $\mathrm{R}$ inferior parietal lobule (BA 40) & 50 & -48 & 47 & CEN \\
\hline R inferior and middle temporal gyrus (BA 20/2I) & 63 & -36 & -15 & CEN \\
\hline R insula, inferior frontal gyrus (BA 14/47) & 32 & 23 & -6 & CEN \\
\hline R middle and superior frontal gyrus (BA 6/8/9/10) & 40 & -35 & 24 & CEN \\
\hline R precuneus (BA 7) & 8 & -70 & 43 & CEN \\
\hline $\mathrm{R}$ cingulate (BA 32)* & 7 & -14 & 29 & CEN \\
\hline R dorsal anterior cingulate cortex/middle frontal gyrus (BA 8/6)* & 7 & 30 & 40 & CEN \\
\hline R angular gyrus/supramarginal gyrus (BA 39/40) & 53 & -55 & 29 & DMN \\
\hline $\mathrm{R}$ middle and inferior temporal gyrus (BA 21/22) & 57 & -10 & -17 & DMN \\
\hline R inferior frontal gyrus (BA 47) & 46 & 29 & -10 & DMN \\
\hline R frontal gyrus (BA I0/9/47) & 12 & 48 & 12 & $\mathrm{DMN}$ \\
\hline R posterior cingulate cortex, precuneus (BA 23/3I/7) & 8 & 52 & 28 & DMN \\
\hline
\end{tabular}

All locations reported are center of mass in MNI coordinates. Global graph metrics are computed across all 5 I nodes. Regions with an asterisk (*) indicate the specific nodes used to examine local graph metrics. 
a

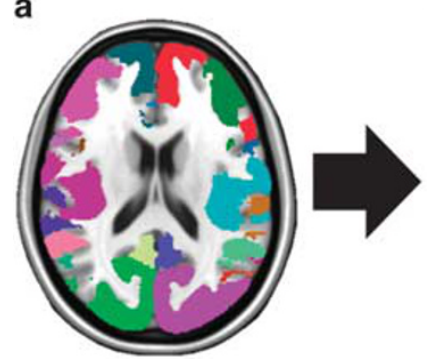

b

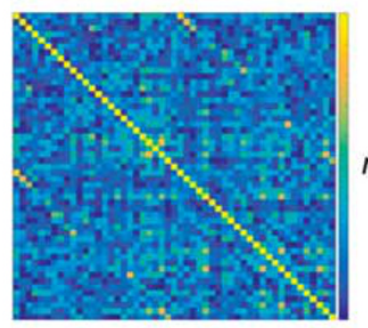

C

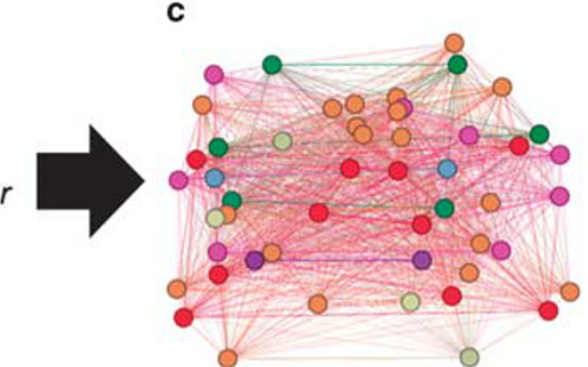

Figure 2 Illustration of graph analysis pipeline. (a) Nodes were defined as the 5 I non-adjacent regions from the Yeo 7-network solution atlas (see Figure I and Table 2 for a summary of the locations of each node). (b) A total of $51 \times 51$ partial correlation adjacency matrices were computed from each pairwise correlation, controlling for the signal from all other regions, for resting-state and task-evoked fMRI data separately, and then thresholded and binarized to create an unweighted adjacency matrix such that all correlations above the threshold will be given a value of I, and below the threshold will be given a value of 0 (see 'Network Binarization' in the Supplementary Information for more details). (c) The resulting $5 \mathrm{I} \times 5 \mathrm{I}$ binarized matrices were then analyzed as whole-brain graphs for each individual for resting-state and task data separately. Graph metrics were then computed to assess global and local network properties (see 'Global and Local Graph Metrics' in the Supplementary Information for more details). Global graph metrics were computed from all $5 \mathrm{I}$ regions, resulting in a single value for each individual for each scan. Local graph metrics were computed from the eight ACC regions for each individual for each scan.

(Rubinov and Sporns, 2010) and in-house scripts in MATLAB (2014a; The MathWorks, Natick, MA). See 'Global and Local Graph Metrics' and Supplementary Table S1 in the Supplementary Information for the equations and descriptions of each metric.

\section{Ineffective Task-Related Suppression of the Default Mode Network}

To replicate previous findings regarding ineffective taskrelated suppression of the DMN in depressed adolescents (Ho et al, 2015), we examined group differences in activation during the response inhibition task within the DMN (Figure 1). See 'Ineffective Task-Related Suppression of the Default Mode Network' in the Supplementary Information for more details.

\section{Group by Brain State Effects in Global Graph Metrics}

For each global graph metric, we conducted a linear mixed effects (LME) model with group (MDD, CTL) and brain state (rest, task) modeled as fixed effects, participant modeled as a random effect, and age, gender, IQ, and motion (defined as the Euclidean norm of the six motion parameters averaged across all time points) included as covariates. All LME models were fit by restricted maximum likelihood (REML) $t$-tests and Sattherthwaite approximations for degrees of freedom provided by $R$ 's lmerTest package (Kuznetsova et al, 2015) were used to compute significance values.

\section{Group by Brain State Effects in Local Graph Metrics}

For each local graph metric in eight-specific ACC nodes of interest (bilateral cingulate gyrus, ACC/superior frontal gyrus, dACC/middle frontal gyrus, orbitofrontal/sgACC), we conducted an LME with group (MDD, CTL) and brain state (rest, task) modeled as fixed effects, participant modeled as a random effect, and age, gender, IQ, and motion included as covariates. All LME models were fit by REML $t$-tests and Sattherthwaite approximations for degrees of freedom were used to compute significance values.

\section{Correction for Multiple Comparisons}

To conservatively account for false-positive inflation from multiple statistical comparisons, we applied Bonferroni correction for the tests of significance in the three local graph metrics from the eight ACC nodes of interest (ie, 24 tests) and the four additional global graph metrics $(\alpha=0.05 / 28=0.0018)$.

\section{Correlations between Graph Metrics and Task Performance}

On the basis of prior work showing that state-dependent changes in the topology of the CEN relate to performance on demanding cognitive tasks in adolescents (Dwyer et al, 2014), we also computed correlations between flexibility of local graph metrics of the ACC and task performance. Specifically, for the local graph metric that showed a significant interaction of group and brain state (see 'Results' section below), we computed the difference in this metric across brain states (rest-task) and correlated this difference value with successful inhibition across all participants. Five MDD and 8 CTL did not have their behavioral responses properly recorded; these 13 individuals were therefore not included in this analysis. A two-tailed Pearson's correlation coefficient test was used for this analysis.

\section{Correlations between Graph Metrics and Clinical Characteristics}

To test whether inflexibly elevated task-evoked functional between the ACC and DMN was associated with greater depression severity and an earlier age of depressive onset (Ho et al, 2015), we computed correlations between the local graph metric that showed a significant interaction of group and brain state (see 'Results' section below) during the task with severity of depression and age of depression onset within the MDD participants only. Severity of depression was measured as total $t$-scores on RADS-2. Age of depression onset was measured as the integer age (in years) that adolescents with MDD reported their first depressive episode occurred. One MDD participant was missing RADS-2 scores 
and five MDD participants did not provide age of onset information; these individuals were not included in their respective analyses. Because of the non-normality of the RADS-2 scores and age of depression onset in the MDD sample, two-tailed Spearman's rank correlation coefficient tests were used for these analyses.

\section{RESULTS}

\section{Demographics}

The groups did not differ significantly in age, gender, pubertal status, socioeconomic status, or IQ (all $p s>0.05$ ). The MDD group endorsed significantly higher levels of depression and anxiety and lower levels of psychosocial function than did the CTL group (all $p s<0.05$ ). See Table 1 for a summary of clinical and demographic information.

\section{Behavioral Results on Response Inhibition Task}

The groups did not differ significantly in mean response time on non-missed GO trials $\left(\mathrm{F}_{1,80}=0.016, p=0.90\right)$. For percentage of successful STOP trials, there was also no main effect of a group $\left(F_{1,80}=0.06, p=0.81\right)$, nor an interaction effect between group and difficulty $\left(F_{1,80}=0.05, p=0.80\right)$. There was a main effect of difficulty $\left(F_{1,80}=155.626\right.$, $p<0.001$ ), with participants successfully stopping more often on EASY trials. Table 1.

\section{Group by Brain State Effects in Global Graph Metrics}

There was no significant main effect for group and no significant interaction of group and brain state for any global graph metric.

\section{Group by Brain State Effects in Local Graph Metrics}

Only local efficiency of the right dorsal anterior cingulate cortex/middle frontal gyrus ( $\mathrm{R} \mathrm{dACC} / \mathrm{MFC}$ ), a region of the CEN (Figure 1), showed significant group by brain state effects after Bonferroni correction $\left(t_{96.08}=3.23 ; p=0.0017\right)$. Post hoc $t$-tests revealed that whereas the MDD group was inflexible in this metric (ie, local efficiency of the R dACC/ MFG did not differ significantly between rest and task), the CTL group exhibited flexibility (ie, local efficiency of $\mathrm{R}$ $\mathrm{dACC} / \mathrm{MFG}$ during task was lower than rest). Figure 3.

\section{Group by Brain State Effects on Weighted Graph Metrics}

As a supplementary analysis, we tested whether there were group by brain state effects in global and local graph metrics computed based on weighted matrices, ie, graphs that have not been thresholded or binarized. We computed all of the metrics with the exception of degree, which is the equivalent as it is for thresholded/binarized matrices, and smallworldness, given controversy regarding generating random weighted matrices and their influence on this metric (Hosseini and Kesler, 2013; Opsahl et al, 2008; Zlatic and Stefancic, 2009). None of these weighted metrics exhibited significant group by brain state interactions (all ps $>0.09$ ). a
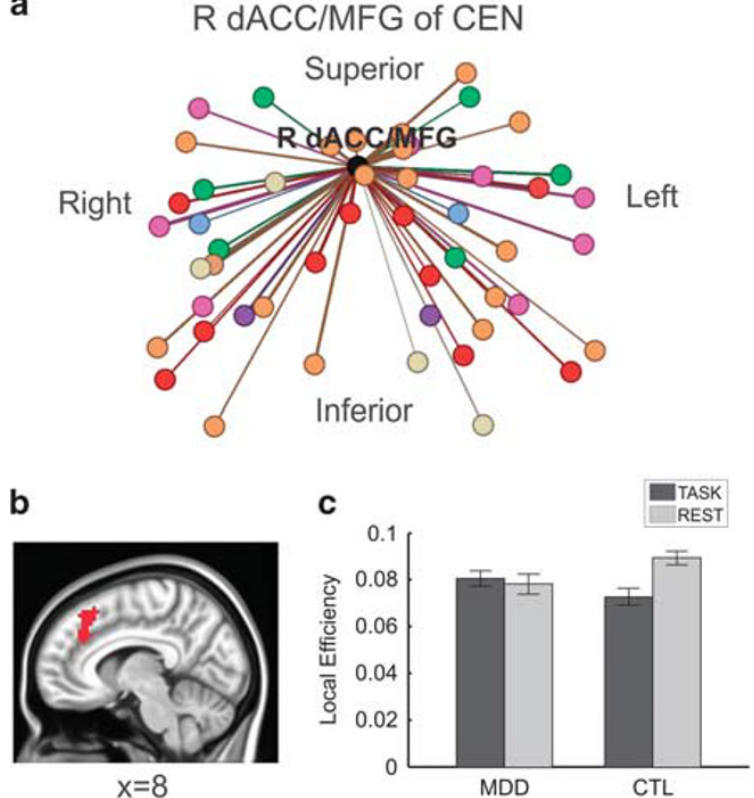

Figure 3 Local efficiency of right dorsal anterior cingulate cortex/middle frontal gyrus ( $\mathrm{R}$ dACC/MFG) showed a significant group difference between task and rest. For global graph metrics, linear mixed effects (LME) models used to assess graph metric showed a significant interaction of group (MDD, CTL) by brain state (REST, TASK); for local graph metrics, LMEs testing group by brain state interaction effects were conducted for each of the 16 nodes of interest. Only local efficiency of the right dACC/MFG was significant after Bonferroni correction. (a) Graph representation of unweighted connections between right dACC/MFG (shown in black) with all other network nodes colored according to network membership (see Figure I) using Gephi (https://gephi.org/). (b) Sagittal view of right dACCl MFG in MNI space (radiological convention). (c) Post hoc t-tests indicated that individuals with MDD did not significantly differ in local efficiency of right $\mathrm{dACC} / \mathrm{MFG}$ between rest and task, whereas the CTL individuals showed lower local efficiency of right dACC/MFG during the task compared to rest.

\section{Testing for Group Differences in Task Activation and Rest in ACC Nodes}

As a supplementary analysis, we tested whether there were group differences in overall task activation, during rest, and in task $v s$ rest in the eight ACC nodes where we focused our local graph analyses. Please see 'Testing for Group Differences in Task Activation in ACC Nodes' in the Supplementary Information and Supplementary Tables S4A, B, C for more details. No ACC regions showed significant group differences in overall task activation (all $p s>0.10$ ), rest (all $p s>0.16$ ), or a significant group by brain state interaction (all $p s>0.19$ ).

\section{Correlations between Graph Metrics and Task Performance}

Adolescents with lower local efficiency of $\mathrm{R}$ dACC/MFG during the task relative to rest (ie, positive flexibility values) performed more poorly on the response inhibition task $(r=-0.228, p=0.039)$. Figure 4 .

\section{Correlations between Graph Metrics and Clinical Characteristics}

Depressed adolescents with lower local efficiency of $\mathrm{R}$ dACC/MFG during the task had an earlier age of MDD 
onset $\left(r_{\mathrm{s}}=0.353, p=0.032\right)$; this metric was not related with depression severity $\left(r_{\mathrm{s}}=0.088, p=0.60\right)$.

\section{DISCUSSION}

This study was designed to test the hypothesis that adolescent MDD reflects altered neurodevelopmental trajectories of ACC connectivity underlying self-regulation, as measured by neural flexibility of network-level topological characteristics. Specifically, we assessed local graph metrics of ACC-based connections among task-positive IFNs during resting state and during a response inhibition task in a cohort of depressed and well-matched non-depressed adolescents. We report three main results. First, depressed adolescents showed inflexibility in local efficiency of right dACC/MFG. Second, although the groups did not differ in behavioral performance, flexibility of right dACC/MFG local efficiency significantly predicted successful performance on the response inhibition task, consistent with recent work indicating that the neural bases of cognitive control depends on the coordinated organization of large-scale task-positive and task-evoked networks (Cole et al, 2013; Dwyer et al, 2014; Shine et al, 2016). Third, depressed adolescents with lower local efficiency in right dACC/MFG during the task reported an earlier age of onset of MDD, which is consistent with the formulation that the ACC has a critical role in adolescent brain development and that early-onset MDD affects typical development (Ho et al, 2015; Lichenstein et al, 2016). Strengths of our study include the application of graph theory to examine how network-level topology and organization changes as a function of brain state (ie, during resting state as compared to during active task processing) in MDD. Given that we found no significant differences between groups in overall task activation or during rest in our ACC regions of interest, our analyses and findings highlight the importance of assessing network-level topology to elucidate the neuropathophysiology of MDD. Furthermore, our study sample of depressed adolescents who are free from potential confounds of chronic disease and medication usage offers potential insight into the processes by which the development of IFNs may go awry in MDD. Importantly, we extend previous work implicating task-related neural inflexibility between ACC and the DMN in depressed adolescents by applying graph theoretical analyses to both resting-state fMRI and task-evoked fMRI in a task assessing cognitive control. Together, our findings support a neurodevelopmental model of ACC connectivity in adolescent MDD, whereby altered typical development of a key region of the $\mathrm{CEN}$ (dACC/MFG), as measured by inflexible local connections, may impact self-regulation processes underlying the pathophysiology of MDD.

Our finding of inflexibility in local efficiency of the dACC/ MFG in depressed adolescents extends the current literature on cognitive control brain regions in MDD (Kaiser et al, 2015a; Miller et al, 2015) and supports the importance of ACC circuitry in adolescent MDD (Lichenstein et al, 2016). The dACC and MFG are involved in the stable maintenance of task representation and behavior, error-related processing, motivational behavior, and the integration of affective stimuli in cognitive control (Bush et al, 2000). Functional and structural abnormalities in ACC and MFG have been

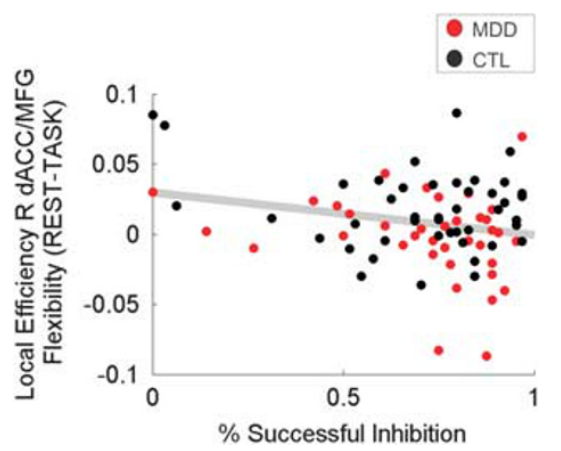

Figure 4 Individual differences in flexibility (ie, rest minus task) of local efficiency of right dorsal anterior cingulate cortex/middle frontal gyrus ( $R$ dACC/MFG) predicted task performance. Lower local efficiency of R dACC/ MFG during the task relative to rest (ie, positive values on the ordinate) was significantly associated with worse inhibition success on the task $\left(r_{82}=-0.228, p=0.039\right)$.

identified in individuals with MDD and are posited to be related to impairments in cognitive and emotional processing domains (Lichenstein et al, 2016). Moreover, connections between nodes in the CEN, including ACC/MFG, are differentially engaged depending on task demands (Braun et al, 2015; Krienen et al, 2014; Kumfor et al, 2015); researchers have recently posited that maladaptive engagement of ACC nodes in task-positive IFNs are likely to underpin psychiatric conditions characterized by impairments in self-regulation, including MDD (Cole et al, 2014b; Kaiser et al, 2015a). Indeed, investigators are increasingly finding that network-level connectivity patterns predict behavioral performance on cognitive control tasks (eg, attention, working memory) and clinical symptoms relevant to self-regulatory processes (eg, ADHD; Giessing et al, 2013; Rosenberg et al, 2016; Sato et al, 2013). Nevertheless, we wish to emphasize that 'neural inflexibility' should not be taken as a blanket term reflecting maladaptive cognitive processes or suboptimal neurodevelopment. It is crucial to note that whether the balance between stable and shifting topological organization of IFNs is adaptive is likely dependent on the specific cognitive process in question (eg, working memory, sustained attention, numerical processing) as well as the developmental stage of the individual (Alavash et al, 2014, 2016; Hutchison and Morton, 2016). We thus speculate that the patterns of neural flexibility promoting adaptive cognitive control may differ from those supporting successful socioemotional regulation, and recommend that future studies incorporate several cognitive and affective tasks to map out these distinctions in adolescents with and without MDD. We further speculate that abnormalities in statedependent dACC/MFG network connectivity may represent transdiagnostic neural markers of self-regulatory difficulties. Future studies are needed to examine these brain-behavior associations across relevant diagnostic groups that span a range of self-regulation difficulties in the realm of cognitive control and socioemotional contexts (eg, ADHD, anxiety).

Although the MDD and healthy comparison groups did not differ significantly in behavioral performance on the task (which gives us confidence that the group differences in state-dependent graph metrics are not driven by group differences in performance), we nonetheless found that 
individual differences in flexibility of dACC/MFG local efficiency predicted behavioral performance across our entire sample. Given previous research that task-related decreases in local efficiency-coupled with task-related increases in global efficiency-are associated with better performance on an attention task (Giessing et al, 2013), it is interesting to note that the healthy comparison group exhibited lower local efficiency of dACC/MFG during the task relative to rest, whereas the MDD group showed limited change in local efficiency of dACC/MFG across brain states. Although speculative, our results may indicate that early MDD alters the development of IFNs that are still maturing during this sensitive period. Indeed, several studies have demonstrated development typical shifts from local to global (or 'distributed') network organization, and that these architectural changes support adaptive higher-level cognition (Fair et al, 2007a; 2009). Ultimately, longitudinal studies in at-risk groups are needed to definitively test these hypotheses.

In our previous investigation examining solely restingstate connectivity, we did not find group differences in local graph measures, perhaps due to the fact that our node definitions were whole IFNs that encompass functionally and anatomically heterogeneous regions (Sacchet et al, 2016). Further, our prior investigation utilized network-based statistics to identify interconnected subnetworks among the whole IFNs - most notably CEN, SN, and DMN-that were hypoconnected in adolescents with MDD compared to never-depressed controls. In contrast, in the present study we applied a parcellation scheme with 51 spatially isolated regions (instead of 17 large-scale networks) that we defined as nodes in our graph analyses, focused on identifying group differences in topological properties of the ACC, and, most critically, investigated whether adolescent MDD is characterized by neural flexibility, or state-dependent changes, in ACC topology. Together, our distinct yet complementary analyses of these data suggest that adolescents with MDD are characterized by large-scale hypoconnectivity among taskpositive and task-negative IFNs, and that inflexibility of a key $\mathrm{CEN}$ hub, the dACC/MFG, may reflect MDD-related selfregulatory processes in this population. How large-scale hypoconnectivity among IFNs, or compromised structural connectivity, underlie flexible shifts among localized regions or hubs to support higher-order cognition remains an active area of research (Behjat et al, 2015; Bolt et al, 2017). Overall, our findings are difficult to compare with other graph studies of resting-state networks in depressed adolescents (Sacchet et al, 2016) and adults (Meng et al, 2014; Zhang et al, 2011) because these studies examined graph metrics computed only from resting-state connectivity, rather than statedependent changes in graph metrics. Nevertheless, our results are consistent with previous research documenting a lack of brain state-dependent changes between task-evoked and intrinsic connectivity among regions in the ACC (notably sgACC and cingulate gyrus) with nodes of the DMN using seed-based functional connectivity methods (Ho et al, 2015).

While no studies have compared network-level topology between undirected and active task engagement in adolescents with MDD, it is important to note that several studies have examined functional connectivity metrics between intask rest (fixation blocks) with periods during active task (Fair et al, 2007b). In contrast to such an approach, we investigated state-dependent changes in IFNs from separate task and resting-state scans. Our approach circumvents potential task spillover effects during fixation and may thus capture signals that are better suited for comparing taskpositive $v s$ task-negative network topology. Indeed, Finn et al (2017) have argued that assessing functional connectivity across brain states enhances meaningful measures of individual variation, and analyzed data from distinct resting-state and several task scans from the Human Connectome Project as a proof of this concept. Furthermore, this approach of comparing brain states measured from independent scans may permit assessments of slower frequency bands at both task and rest, as such analyses would not be limited to the duration of fixation (or active task) blocks, and may be better suited for investigators interested in examining the contribution of specific frequency bands on network topology or how variability unfolds in these signals within a scan or over time.

Although this is the first study to use graph analysis to assess both task-positive and task-negative connectivity of the ACC in MDD, the relatively narrow age range of our participants limits the generalizability of our findings to younger or older individuals with MDD. Further, our crosssectional design limits our understanding of the possible causal role that neural inflexibility of the dACC/MFG plays in the development of MDD. Research designs with participants across a larger age range are needed to test if our findings generalize to child and adult samples of MDD. Greater variability in clinical characteristics would also permit the assessment of varying ages of onset, chronicity, and illness duration with neural flexibility of the dACC/MFG in the context of self-regulatory processes. Another limitation of our study is the lack of group-related behavioral differences on the task. Our brain-behavior correlations must therefore be interpreted with caution. Future studies implementing cognitive control tasks that elicit sufficient variability in task performance and that detect differences between diagnostic and control groups are needed to extend and replicate our findings. It must also be noted that our current results may be influenced by specific frequency bands and the correlation and density structure of the functional networks. Given evidence that task-evoked connectivity tends to be greater across the fullband, for consistency we conservatively applied the same filter to both sets of data, as has been done in previous studies comparing task-based and resting-state connectivity (Dwyer et al, 2014; Goparaju et al, 2014). Future research is thus needed to systematically assess contributions of specific frequencies and network density/correlation strength to network inflexibility more generally, as well as in the context of disorders such as MDD. Finally, our study assessed graph metrics derived from time-resolved rather than time-varying (herein termed 'dynamic') changes in functional connectivity. Recent investigations in adults with MDD have documented patterns of increased variability in $\mathrm{SN}$, but decreased variability in DMN (Kaiser et al, 2015b). Moreover, there is evidence that over development, large-scale networks become more variable in their coordination at rest, but less variable in their coordination during tasks (Hutchison and Morton, 2016). The correspondence between statedependent dynamics and flexibility of network properties based on static signals is still unknown. Future work 
incorporating sliding window analyses (Kaiser et al, 2015b) or multiplication of temporal derivatives (Shine et al, 2015) on both task-based and resting-state fMRI data are needed to clarify these relationships. Additional longitudinal research is also needed to understand how neural flexibility in specific circuits changes across typical development and how such changes increase risk for MDD during adolescence.

In summary, this study is the first to use network science to assess topological connectivity changes of the ACC across brain states in order to characterize MDD from a neurodevelopmental perspective. Our findings suggest that depressed adolescents show inflexibility in network engagement of the dACC/MFG, as measured by limited changes in local efficiency between rest and active task processing. Building from current evidence on maturational processes in ACC connectivity during adolescence, our results fill a significant gap in our understanding of the neuropathophysiology of depression by providing evidence that MDD alters (or reflects altered) development of IFNs that are still maturing during this sensitive developmental period and by offering potential biomarkers in the form of quantifiable network-based metrics. Validating these findings in longitudinal studies across a wider range of development promises to advance the field by characterizing the typical development of cognitive control networks and by determining if state-dependent dACC/MFG connectivity can be used to identify individuals at risk for MDD, monitor disease progression, and track or predict symptom changes in response to treatments targeting ACC-based circuitry.

\section{FUNDING AND DISCLOSURE}

This work was supported by the American Foundation for Suicide Prevention (PDF-1-064-13 to TCH), the National Science Foundation (IGERT Award 080700 and Graduate Research Fellowship Award DGE-1147470 to MDS), the National Institutes of Health (R01MH085734 to TTY; R01MH101495 to IHG; R01HD072074 to OT; R21AT0 09173 to OT and TTY), the UCSF Research Evaluation and Allocation Committee (to OT and TTY), the J Jacobson Fund (to OT and TTY), the Center of Excellence in Stress and Mental Health and Veteran's Affairs (I-1-CX000715 to ANS), and the Brain and Behavior Research Foundation (Young Investigator Award to TTY). The authors declare no conflict of interest.

\section{ACKNOWLEDGMENTS}

We wish to thank Jing (James) Wu, Kevin Han, Audrey Fortezzo, and Nisreen Mobayed for their help with data collection; Vinod Menon, Mac Shine, and Pat Bissett for helpful discussions; and to the participants and their families for their contribution to our research.

\section{REFERENCES}

Alavash M, Doebler P, Holling H, Thiel CM, Giessing C (2014). Is functional integration of resting state brain networks an unspecific biomarker for working memory performance? Neuroimage 108: 182-193.
Alavash M, Thiel CM, Giessing C (2016). Dynamic coupling of complex brain networks and dual-task behavior. Neuroimage 129: 233-246.

Behjat H, Leonardi N, Sornmo L, Van De Ville D (2015). Anatomically-adapted graph wavelets for improved group-level fMRI activation mapping. Neuroimage 123: 185-199.

Blakemore SJ, Choudhury S (2006). Development of the adolescent brain: implications for executive function and social cognition. J Child Psychol Psychiatry 47: 296-312.

Bolt T, Nomi JS, Rubinov M, Uddin LQ (2017). Correspondence between evoked and intrinsic functional brain network configurations. Hum Brain Mapp 38: 1992-2007.

Braun U, Schafer A, Walter H, Erk S, Romanczuk-Seiferth N, Haddad L et al (2015). Dynamic reconfiguration of frontal brain networks during executive cognition in humans. Proc Natl Acad Sci USA 112: 11678-11683.

Bush G, Luu P, Posner MI (2000). Cognitive and emotional influences in anterior cingulate cortex. Trends Cogn Sci 4: 215-222.

Cole MW, Bassett DS, Power JD, Braver TS, Petersen SE (2014a). Intrinsic and task-evoked network architectures of the human brain. Neuron 83: 238-251.

Cole MW, Repovs G, Anticevic A (2014b). The frontoparietal control system: a central role in mental health. Neuroscientist 20: 652-664.

Cole MW, Reynolds JR, Power JD, Repovs G, Anticevic A, Braver TS (2013). Multi-task connectivity reveals flexible hubs for adaptive task control. Nat Neurosci 16: 1348-1355.

Connolly CG, Wu J, Ho TC, Hoeft F, Wolkowitz O, Eisendrath S et al (2013). Resting-state functional connectivity of subgenual anterior cingulate cortex in depressed adolescents. Biol Psychiatry 74: 898-907.

Cox RW (1996). AFNI: software for analysis and visualization of functional magnetic resonance neuroimages. Comput Biomed Res 29: $162-173$.

Davey CG, Harrison BJ, Yucel M, Allen NB (2012). Regionally specific alterations in functional connectivity of the anterior cingulate cortex in major depressive disorder. Psychol Med 42: 2071-2081.

Davey CG, Whittle S, Harrison BJ, Simmons JG, Byrne ML, Schwartz OS et al (2014). Functional brain-imaging correlates of negative affectivity and the onset of first-episode depression. Psychol Med 45: 1001-1009.

Dodds CM, Morein-Zamir S, Robbins TW (2011). Dissociating inhibition, attention, and response control in the frontoparietal network using functional magnetic resonance imaging. Cereb Cortex 21: 1155-1165.

Dwyer DB, Harrison BJ, Yucel M, Whittle S, Zalesky A, Pantelis C et al (2014). Large-scale brain network dynamics supporting adolescent cognitive control. J Neurosci 34: 14096-14107.

Fair DA, Cohen AL, Power JD, Dosenbach NU, Church JA, Miezin FM et al (2009). Functional brain networks develop from a 'local to distributed' organization. PLoS Comput Biol 5: e1000381.

Fair DA, Dosenbach NU, Church JA, Cohen AL, Brahmbhatt S, Miezin FM et al (2007a). Development of distinct control networks through segregation and integration. Proc Natl Acad Sci USA 104: 13507-13512.

Fair DA, Schlaggar BL, Cohen BAA, Miezin FM, Dosenbach NU, Wenger KK et al (2007b). A method for using blocked and eventrelated FMRI data to study "resting state" functional connectivity. Neuroimage 35: 396-405.

Finn ES, Scheinost D, Finn DM, Shen X, Papademetris X, Constable RT (2017). Can brain state be manipulated to emphasize individual differences in functional connectivity? Neuroimage (doi:10.1016/j. neuroimage.2017.03.064).

Fox MD, Snyder AZ, Vincent JL, Corbetta M, Van Essen DC, Raichle ME (2005). The human brain is intrinsically organized into dynamic, anticorrelated functional networks. Proc Natl Acad Sci USA 102: 9673-9678. 
Giessing C, Thiel CM, Alexander-Bloch AF, Patel AX, Bullmore ET (2013). Human brain functional network changes associated with enhanced and impaired attentional task performance. J Neurosci 33: 5903-5914.

Goparaju B, Rana KD, Calabro FJ, Vaina LM (2014). A computational study of whole-brain connectivity in resting state and task fMRI. Med Sci Monit 20: 1024-1042.

Hamilton JP, Farmer M, Fogelman P, Gotlib IH (2015). Depressive rumination, the default-mode network, and the dark matter of clinical neuroscience. Biol Psychiatry 78: 224-230.

Ho TC, Connolly CG, Henje Blom E, LeWinn KZ, Strigo IA, Paulus MP et al (2014). Emotion-dependent functional connectivity of the default mode network in adolescent depression. Biol Psychiatry 78: 635-646.

Ho TC, Connolly CG, Henje Blom E, LeWinn KZ, Strigo IA, Paulus MP et al (2015). Emotion-dependent functional connectivity of the default mode network in adolescent depression. Biol Psychiatry 78: 635-646.

Hosseini SM, Kesler SR (2013). Influence of choice of null network on small-world parameters of structural correlation networks. PLOS ONE 8: e67354.

Hutchison RM, Morton JB (2016). It's a matter of time: reframing the development of cognitive control as a modification of the brain's temporal dynamics. Dev Cogn Neurosci 18: 70-77.

Jacobs RH, Jenkins LM, Gabriel LB, Barba A, Ryan KA, Weisenbach SL et al (2014). Increased coupling of intrinsic networks in remitted depressed youth predicts rumination and cognitive control. PLoS ONE 9: e104366.

Jin C, Gao C, Chen C, Ma S, Netra R, Wang Y et al (2011). A preliminary study of the dysregulation of the resting networks in first-episode medication-naive adolescent depression. Neurosci Lett 503: 105-109.

Kaiser RH, Andrews-Hanna JR, Wager TD, Pizzagalli DA (2015a). Large-scale network dysfunction in major depressive disorder: a meta-analysis of resting-state functional connectivity. JAMA Psychiatry 72: 603-611.

Kaiser RH, Whitfield-Gabrieli S, Dillon DG, Goer F, Beltzer M, Minkel J et al (2015b). Dynamic resting-state functional connectivity in major depression. Neuropsychopharmacology 41: 1822-1830.

Kelly AM, Di Martino A, Uddin LQ, Shehzad Z, Gee DG, Reiss PT et al (2009). Development of anterior cingulate functional connectivity from late childhood to early adulthood. Cereb Cortex 19: 640-657.

Kessler RC, Berglund P, Demler O, Jin R, Merikangas KR, Walters EE (2005). Lifetime prevalence and age-of-onset distributions of DSM-IV disorders in the National Comorbidity Survey Replication. Arch Gen Psychiatry 62: 593-602.

Krienen FM, Yeo BT, Buckner RL (2014). Reconfigurable task-dependent functional coupling modes cluster around a core functional architecture. Phil Trans R Soc B 369: 20130526.

Kumfor F, Dermody N, Irish M (2015). Considering the impact of large-scale network interactions on cognitive control. J Neurosci 35: $1-3$.

Kuznetsova A, Brockhoff PB, Christensen RHB (2015). Package 'ImerTest'. $R$ package version: 2.0-29 http://cran.uib.no/web/ packages/lmerTest/lmerTest.pdf.

Lichenstein SD, Verstynen T, Forbes EE (2016). Adolescent brain development and depression: a case for the importance of connectivity of the anterior cingulate cortex. Neurosci Biobehav Rev 70: 271-287.

Lucas CP, Zhang H, Fisher PW, Shaffer D, Regier DA, Narrow WE et al (2001). The DISC Predictive Scales (DPS): efficiently screening for diagnoses. J Am Acad Child Adolesc Psychiatry 40: 443-449.

Margulies DS, Kelly AM, Uddin LQ, Biswal BB, Castellanos FX, Milham MP (2007). Mapping the functional connectivity of anterior cingulate cortex. Neuroimage 37: 579-588.
Meng C, Brandl F, Tahmasian M, Shao J, Manoliu A, Scherr M et al (2014). Aberrant topology of striatum's connectivity is associated with the number of episodes in depression. Brain 137(Pt 2): 598-609.

Menon V (2011). Large-scale brain networks and psychopathology: a unifying triple network model. Trends Cogn Sci 15: 483-506.

Miller CH, Hamilton JP, Sacchet MD, Gotlib IH (2015). Metaanalysis of functional neuroimaging of major depressive disorder in youth. JAMA Psychiatry 72: 1045-1053.

Opsahl T, Colizza V, Panzarasa P, Ramasco JJ (2008). Prominence and control: the weighted rich-club effect. Phys Rev Lett 101: 168702.

Power JD, Fair DA, Schlaggar BL, Petersen SE (2010). The development of human functional brain networks. Neuron 67: 735-748.

Reynolds WM. Reynolds adolescent depression scale. John Wiley \& Sons, Inc.; 2010.

Rosenberg MD, Finn ES, Scheinost D, Papademetris X, Shen X, Constable RT et al (2016). A neuromarker of sustained attention from whole-brain functional connectivity. Nat Neurosci 19: 165-171.

Rubinov M, Sporns O (2010). Complex network measures of brain connectivity: uses and interpretations. Neuroimage 52: 1059-1069.

Sacchet MD, Ho TC, Connolly CG, Tymofiyeva O, Lewinn KZ, Han LK et al (2016). Large-scale hypoconnectivity between resting-state functional networks in unmedicated adolescent major depressive disorder. Neuropsychopharmacology 41: 2951-2960.

Sato JR, Takahashi DY, Hoexter MQ, Massirer KB, Fujita A (2013). Measuring network's entropy in ADHD: a new approach to investigate neuropsychiatric disorders. Neuroimage 77: 44-51.

Seeley WW, Menon V, Schatzberg AF, Keller J, Glover GH, Kenna $\mathrm{H}$ et al (2007). Dissociable intrinsic connectivity networks for salience processing and executive control. J Neurosci 27: 2349-2356.

Shaffer D, Fisher P, Lucas CP, Dulcan MK, Schwab-Stone ME (2000). NIMH Diagnostic Interview Schedule for Children Version IV (NIMH DISC-IV): description, differences from previous versions, and reliability of some common diagnoses. J Am Acad Child Adolesc Psychiatry 39: 28-38.

Shine JM, Bissett PG, Bell PT, Koyejo O, Balsters JH, Gorgolewski KJ et al (2016). The dynamics of functional brain networks: integrated network states during cognitive task performance. Neuron 92: 544-554.

Shine JM, Koyejo O, Bell PT, Gorgolewski KJ, Gilat M, Poldrack RA (2015). Estimation of dynamic functional connectivity using multiplication of temporal derivatives. Neuroimage 122: 399-407.

Smith SM, Jenkinson M, Woolrich MW, Beckmann CF, Behrens TE, Johansen-Berg $\mathrm{H}$ et al (2004). Advances in functional and structural MR image analysis and implementation as FSL. Neuroimage 23(Suppl 1): S208-S219.

Sole-Padulles C, Castro-Fornieles J, de la Serna E, Calvo R, Baeza I, Moya $J$ et al (2016). Intrinsic connectivity networks from childhood to late adolescence: effects of age and sex. Dev Cogn Neurosci 17: 35-44.

Strikwerda-Brown C, Davey CG, Whittle S, Allen NB, Byrne ML, Schwartz OS et al (2015). Mapping the relationship between subgenual cingulate cortex functional connectivity and depressive symptoms across adolescence. Soc Cogn Affect Neurosci 10: 961-968.

Uddin LQ (2014). Salience processing and insular cortical function and dysfunction. Nat Rev Neurosci 16: 55-61.

Uddin LQ, Supekar KS, Ryali S, Menon V (2011). Dynamic reconfiguration of structural and functional connectivity across core neurocognitive brain networks with development. J Neurosci 31: $18578-18589$. 
Yeo BT, Krienen FM, Sepulcre J, Sabuncu MR, Lashkari D, Hollinshead $\mathrm{M}$ et al (2011). The organization of the human cerebral cortex estimated by intrinsic functional connectivity. J Neurophysiol 106: 1125-1165.

Zhang J, Wang J, Wu Q, Kuang W, Huang X, He Y et al (2011). Disrupted brain connectivity networks in drug-naive, firstepisode major depressive disorder. Biol Psychiatry 70: 334-342.
Zheng H, Xu L, Xie F, Guo X, Zhang J, Yao L et al (2015). The altered triple networks interaction in depression under resting state based on graph theory. Biomed Res Int 2015: 386326.

Zlatic V, Stefancic H (2009). Influence of reciprocal edges on degree distribution and degree correlations. Phys Rev E Stat Nonlin Soft Matter Phys 80(1 Pt 2): 016117.

Supplementary Information accompanies the paper on the Neuropsychopharmacology website (http://www.nature.com/npp) 